\title{
Trastorno de estrés post traumático en niños y adolescentes
}

\author{
FLORA DE LA BARRA Mac D. ${ }^{1}$
}

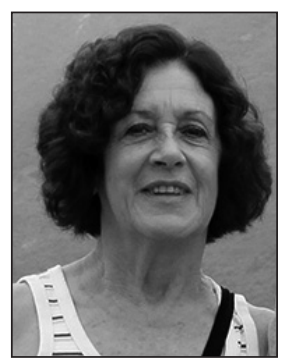

1. Médico psiquiatra de niños y adolescentes. Profesor Adjunto Depto. Salud Mental Oriente Facultad de Medicina. Universidad de Chile. Departamento de Psiquiatría Clínica las Condes.

Los estudios de seguimiento de cohortes muestran que entre el $25 \%$ y el $60 \%$ de los niños ha sufrido un evento traumático significativo antes de llegar a la adultez. Los traumas pueden ser domésticos (maltrato, abuso sexual), comunitarios, violencia escolar, desastres naturales, accidentes, médicos, guerra, terrorismo, asociados al status de refugiados, etc. Sin embargo, la mayoría de los niños son resilientes frente al trauma. Los diversos estudios internacionales muestran prevalencias de Trastorno de Estrés post Traumático (TEPT) entre 6 y 13\%, sin embargo, los análisis por tipo de trauma muestran que las tasas de TEPT relacionadas con desastres naturales son más altas, $40-90 \%$. Por otra parte, los niños que tienen síntomas sub-sindromáticos, presentan impedimento funcional comparable a los que cumplen criterios diagnóstico para TEPT ${ }^{1-4}$. Con el tiempo, las tasas de TEPT disminuyen, pero persisten en algunos grupos ${ }^{5}$.

El trastorno por estrés agudo aparece como consecuencia de la exposición al evento traumático y puede tener distintos niveles de intensidad hasta 3 meses después de ocurrido.

E1 TEPT se puede entender como una falla de la recuperación. Aparece meses después de que la persona ha sufrido un evento significativo e impactante. El diagnóstico sólo puede efectuarse a partir de un mes después de ocurrido. Antes podría tratarse de síntomas nor- mativos, reacción aguda al estrés o reacción adaptativa.

Los criterios diagnósticos señalados para adultos no siempre están presentes en los niños. Los niños pequeños no reportan las vivencias de re-experimentación del trauma; en vez de eso lo actúan, o temas o aspectos relacionados en juegos repetitivos o relatan sueños terroríficos sin contenido específico del trauma. Sufren intenso malestar fisiológico o psicológico al estar frente a recordatorios del hecho (personas, lugares, situaciones, estímulos), sin que hablen de ello. Se observan síntomas de hiperactivación como dificultad para quedarse dormido, irritabilidad o crisis de rabia, dificultad de concentración o reacción de sobresalto aumentada. Igualmente pueden presentarse síntomas asociados como agresividad, oposicionismo, regresión en su desarrollo (control esfínteres, lenguaje) y miedos no relacionados con el trauma. También es frecuente una disminución del rendimiento académico, del coeficiente intelectual, de las funciones ejecutivas, atención y memoria ${ }^{6}$.

Son predictores de riesgo la exposición a traumas previos, traumas múltiples, mayor exposición al trauma índice, trastorno psiquiátrico preexistente, especialmente trastornos ansiosos, psicopatología parental, adversidad familiar y falta de apoyo social. En el contexto de un desastre, ver eventos relacionados por 
televisión, evacuación retrasada del lugar, experimentar síntomas extremos de pánico, sentir su vida o la de un ser querido en peligro.

Constituyen factores protectores el apoyo parental, niveles bajos de TEPT parental, y también ayuda que los padres hayan tenido sintomatología leve o hayan resuelto sus propios síntomas.

Es frecuente la comorbilidad con trastorno de ansiedad, Depresión, Deficit de Atención e Hiperactividad y abuso de sustancias.

Los estudios neurobiológicos en niños que sufrieron trauma y TEPT comparados con los que no lo desarrollaron, muestran que el estrés temprano puede llevar a una desregulación permanente del eje Hipotálamo-Hipófisis-Adrenal, que a su vez predispone a vulnerabilidad psiquiátrica a lo largo de la vida. Se han encontrado alteraciones anatómicas y funcionales del cerebro en desarrollo como disminución de volumen del cuerpo calloso, cerebelo y de sustancia gris en la corteza prefrontal (CPF). En adolescentes se ha observado alteraciones de la activación de la CPF mediante Resonancia Magnética funcional.

Algunas variantes genéticas (transportador 5HTT, alelo MET de BDNF, polimorfismo MAOA y COMT) agregan pequeños aumentos en el riesgo, interactuando con el trauma, dependiendo del programa evolutivo que está ocurriendo en ese momento. En los períodos críticos de plasticidad aumentada los niños son especialmente vulnerables, ya que aumenta la probabilidad que estresores posteriores induzcan síntomas o trastornos. Otros genotipos pueden ser predictores de resiliencia, junto a moderadores ambientales ${ }^{8-10}$.

Respecto al tratamiento, existen recomendaciones basadas en la evidencia. Las terapias más eficaces están centradas en el trauma: abordan directamente las experiencias traumáticas del niño, incorporan a los padres al tratamiento y se focalizan no sólo en mejoría de síntomas, sino también en el funcionamiento, resiliencia y trayectoria evolutiva. Hay 7 estudios controlados que demuestran la efectividad de la Terapia Conductual Cognitiva focalizada en el trauma ${ }^{11-13}$.

Cuando se necesita una reducción aguda de los síntomas, o se comprueba un trastorno comórbido o la respuesta a psicoterapia es parcial, está indicado agregar farmacoterapia. Las evidencias de eficacia más importantes apoyan el uso de antidepresivos ISRS. El propanolol administrado inmediatamente después del trauma, disminuye los síntomas de re-experiencia ${ }^{14}$.

Las investigaciones están demostrando que es posible modificar los efectos deletéreos psicológicos y biológicos del estrés temprano mediante programas de enriquecimiento ambiental e intervenciones psicosociales. Después que ocurren traumas comunitarios, es útil hacer tamizaje en escuelas y otros ambientes donde se reúnen los niños, comenzando después de 1 mes. También es necesario pesquisar factores de riesgo conocidos para evitar secuelas posteriores. Existen manuales de apoyo para padres, profesores y profesionales de atención primaria, que entregan estrategias para intervenir en la etapa aguda post desastre y por el tiempo que sea necesario. Se han diseñado programas promotores de la resiliencia indicados para inmunizar a los niños frente a los efectos adversos de hechos traumáticos ${ }^{15}$.

Este número especial de la Revista Chilena de Pediatría está dedicado a analizar los problemas emocionales/conductuales y los trastornos psiquiátricos en niños chilenos después del trauma agudo colectivo causado por el terremoto y tsunami del 27 Febrero 2010. Se analizan también los traumas y las secuelas psiquiátricas a más largo plazo. Dos estudios realizados en niños afectados de las regiones del Maule y del Bío Bío utilizando distintas medidas dimensionales, encuentran altas prevalencias de TEPT (30\% y 40\%) 6-8 meses después del terremoto y señalan los distintos factores de riesgo.

En el tercer trabajo, se estudiaron los efectos del trauma sobre los cuidadores de niños consultantes al sistema de salud, detectando un 7\% de TEPT auto-reportado. Los 3 estudios entregan valiosa información de nuestra realidad y señalan que los efectos del desastre sobre la salud mental de nuestros niños y sus familias continúan manifestándose.

El último trabajo informa sobre un programa de intervención multimodal en una comuna de la VII región. Aunque en la mayoría de los 
casos se trataba de psicopatología preexistente, y muy pocos se habían agravado post terremoto, son destacables los aspectos de prevención secundaria en la red de salud y educación del lugar.

\section{Referencias}

1.- Alisic E, van de Schoot T, van Ginkel J, Kleber R: Looking Beyond Posttraumatic Stress Disorder in Children: Posttraumatic Stress Reactions, Posttraumatic Growth and Quality of Life in a General Population Sample. J Clin Psychiatry 2008; 69 (9): 1455-61.

2.- Copeland W, Keeler G, Angold A, Costello J: Traumatic events and Posttraumatic Stress in Childhood. Arch Gen Psychiatry 2007; 64: 577-84.

3.- Cozza S, Guimond J: Children and Adolescents. In Benedek D \% Wynn G. Clinical manual for management of PTSD. American Psychiatric Publishing 2011; 291 323.

4.- McLaughlin K A, Fairbank $J$ A, Gruber $M J$ et al: Serious emotional disturbance among youths exposed exposed to hurricane Katrina 2 years post disaster. J. Am Acad Child Adolesc Psychiatry 2009; 48: 1069-78.

5.- Scheeringa M, Zeanah C, Myers L, Putnam F: Predictive Validity in a Prospective Follow-up of PTSD in Preschool Children. J Am Acad Child Adolesc Psychiatry 2005; 44 (9): 899-906.

6.- De Bellis M, Van Dillen T: Childhood Post-Traumatic Stress Disorder: An Overview. Child and Adolescent Psychiatric Clinics of North America 2005; 14 (4): 745 72.

7.- Carrion VG, Weems CF, Ray R, Reiss AR: Toward an
Empirical Definition of Pediatric PTSD in Youth. J Am Acad Child Adolesc Psychiatry 2002; 41 (2): 166-73.

8.- Martin E, Ressler K, Binder E, Nemeroff C: The Neurobiology of Anxiety Disorders: Brain Imaging, Genetics and Psychoneuroendocrinology. Psych. Clin N Am 2009; 32: 549-575.

9.- Teicher M, Andersen S, Polcari A, Anderson C, Navalta $C$ : Developmental Neurobiology of Childhood Stress and Trauma. Psychiatr Clin N Am 2002; 25 (2): $397-$ 426.

10.- Jacowsky AP, de Araujo CM, Tavares de Lacerda AL, Mari JJ, Kaufman J: Neurostructural Imaging Findings in Children with Post-traumatic Stress Disorder: Brief Review. Psychiatry and Clinical Neurosciences 2009; 63: 1-8.

11.- AACAP Official Action: Practice parameter for the assesment and treatment of children and adolescents with Post Traumatic Stress Disorder. J Am Acad Child Adolesc Psychiatry 2010; 49 (4): 414-30.

12.- Pine D, Cohen J: Trauma in Children and Adolescents: Risk and treatment of Psychiatric Sequelae. Biol Psychiatry 2002; 51: 519-531.

13.- Cohen J, Mannarino A: Trauma-focused Cognitive Behavioral Therapy for children and Parents. Child \& Adolescent Mental Health 2008; 13: 158-62.

14.- Davidson J: Pharmacologic treatment of Acute and Chronic Stress Following Trauma: 2006. J Clin Psychiatry 2006; 67 (suppl 2): 34-9.

15.- Hoagwood KE, Vogel J, Levitt JM, D’ Amico P, Paisner $W$, Kaplan S: Implementing an Evidence-Based Trauma Treatment in a State System After September 11: the CATS Project. J Am Acad Child \& Adolesc Psychiatry 2007; 46 (6) 773-9. 